\title{
Multi CVD coated layers and their effect on the performance of cutting tools
}

\section{R. PORAT}

ISCAR Ltd., Tefen, Israel

\begin{abstract}
In this paper we represent tool life of C.V.D. coated cutting tools, in machining steel with different cutting speeds and comparison between coated cutting tools with multi coated layers, to single layers.

While we increased the number of coating layers to achieve the same coating thickness we got the advantage of higher tool life in machining steels. However, aside from having higher wear resistance, using this combination also helps us to increase efficiency of the machining process by increasing the cutting speed.
\end{abstract}

The explanation for this advantage of wear resistance and the capability to withstand high cutting speed can be the multi layer interuptions, as these interuptions of the wear mechanism cause a halt in the wear procedure.

This multi coating layer can reduce and even neglect the effect of the substrate properties on the behaviour of the coating, i.e., this combination is the best diffusion barrier for cobalt and carbon from the substrate to the coating, so the properties of the coating are unaffected by the properties of the cemented carbide subtrate.

Another advantage is that while in single layers, increasing the thickness of the coating causes a reduction of the good mechanical properties, such as transverse rupture strength, and increases the sensivity for fractures. In this case, the multi layer coating behaved like a stopping mechanism to eliminate propagation of the cracks, so that the thick coating behaved like a thin coating enabling all the mechanical properties of the substrate to remain with only small reductions in the mechanical properties.

By using multi layers we can increase the rate of deposition. In the beginning of every coating process the rate of deposition is extremely high, after which it gradually reaches a much slower rate. By starting each layer from the beginning, and having higher amounts of layers, we succeed to increase the deposition rate, and achieve greater efficiency in the crystal growth rate. 